\title{
Ultrafast Enhancement of Ferromagnetic Spin Exchange Induced by Ligand-to-Metal Charge Transfer
}

\author{
A. Ron, ${ }^{1,2,3}$ S. Chaudhary, ${ }^{1,2}$ G. Zhang, ${ }^{4}$ H. Ning, ${ }^{1,2}$ E. Zoghlin $\odot,{ }^{5}$ S. D. Wilson, ${ }^{5}$ R. D. Averitt $\odot,{ }^{4}$ \\ G. Refael, ${ }^{1,2}$ and D. Hsieh $\oplus^{1,2, *}$ \\ ${ }^{1}$ Department of Physics, California Institute of Technology, Pasadena, California 91125, USA \\ ${ }^{2}$ Institute for Quantum Information and Matter, California Institute of Technology, Pasadena, California 91125, USA \\ ${ }^{3}$ Raymond and Beverly Sackler School of Physics and Astronomy, Tel-Aviv University, Tel Aviv, 69978, Israel \\ ${ }^{4}$ Department of Physics, University of California, San Diego, La Jolla, California 92093, USA \\ ${ }^{5}$ Materials Department, University of California, Santa Barbara, Santa Barbara, California 93106, USA
}

(Received 10 December 2019; revised 17 August 2020; accepted 2 October 2020; published 4 November 2020)

\begin{abstract}
We theoretically predict and experimentally demonstrate a nonthermal pathway to optically enhance superexchange interaction energies in a material based on exciting ligand-to-metal charge-transfer transitions, which introduces lower-order virtual hopping contributions that are absent in the ground state. We demonstrate this effect in the layered ferromagnetic insulator $\mathrm{CrSiTe}_{3}$ by exciting $\mathrm{Te}-\mathrm{to}-\mathrm{Cr}$ charge-transfer transitions using ultrashort laser pulses and detecting coherent phonon oscillations that are impulsively generated by superexchange enhancement via magneto-elastic coupling. This mechanism kicks in below the temperature scale where short-range in-plane spin correlations begin to develop and disappears when the excitation energy is tuned away from the charge-transfer resonance, consistent with our predictions.
\end{abstract}

DOI: 10.1103/PhysRevLett.125.197203

Directly modifying spin exchange energies in a magnetic material with light can enable ultrafast nonthermal control of its magnetic states. Such a capability could potentially provide a pathway to engineer thermally inaccessible spin Hamiltonians, study out-of-equilibrium quantum manybody phenomena, and realize high-speed information processing technologies. Current approaches rely on tuning charge hopping amplitudes that mediate exchange interactions by optically exciting either virtual [1-9] or real [10-13] charge-transfer (CT) transitions between magnetic sites. In this Letter, we show that when an exchange interaction is mediated by a nonmagnetic ligand, optically exciting a real CT transition from the ligand to magnetic site enhances the exchange potentially severalfold by introducing lower order virtual hopping contributions. Using a phase-resolved coherent phonon spectroscopy technique, we demonstrate this mechanism in $\mathrm{CrSiTe}_{3}$, a prototypical superexchange dominated ferromagnetic insulator.

In superexchange dominated magnetic insulators, as is realized in many transition metal oxide and chalcogenide based materials, the superexchange energy $J_{\text {ex }}$ between spins on neighboring metal sites depends on the number of virtual hopping processes that mediate the interaction. A simple illustrative model of ferromagnetic superexchange consists of two metal ions, each with a singly occupied $d$ orbital, interacting via a ligand ion with fully occupied $p_{x}$ and $p_{y}$ orbitals in a $90^{\circ}$ geometry [Fig. 1(a)]. To leading order in perturbation theory $J_{\mathrm{ex}} \propto-\left(t / \Delta_{\mathrm{CT}}\right)^{4} J_{H}$, where $t$ is the metal-to-ligand hopping energy, $\Delta_{\mathrm{CT}}$ is the charge transfer energy between the ligand $p$ and metal $d$ orbital, and $J_{H}$ is the Hund's coupling between the $p$ orbitals on the ligand site [14]. This expression reflects the fact that the

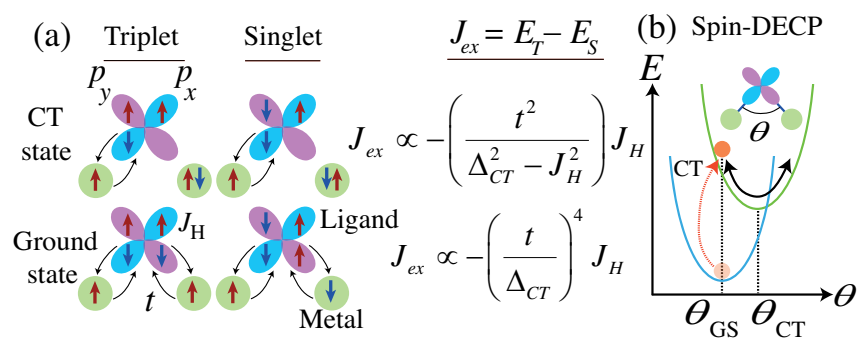

FIG. 1. Ultrafast exchange enhancement induced displacive excitation of coherent phonons (spin-DECP). (a) Schematic showing the triplet and singlet spin configurations of our toy model before (bottom row) and after (top row) CT excitation. The superexchange energy $J_{\mathrm{ex}}$, defined as the triplet and singlet energy splitting, is a function of the metal $d$ orbital to ligand $p_{x, y}$ orbital hopping energy $t$, the charge-transfer energy $\Delta_{\mathrm{CT}}$, and the Hund's coupling $J_{H}$ between the ligand $p$ orbitals. Owing to the bond angle $\theta$ being near $90^{\circ}$, hopping from the left (right) metal site to the $p_{y}\left(p_{x}\right)$ ligand orbital is neglected. Expressions for $J_{\mathrm{ex}}$ derived from a perturbative calculation [14] in both the ground state (GS) and CT state are displayed to the right. (b) Schematic of the potential energy landscape of the nuclei as a function of $\theta$. The CT excitation (dotted red line) causes a sudden shift in the potential minimum due to an impulsive change in $J_{\text {ex }}$, launching coherent phonon oscillations about the new minimum. 
superexchange is ferromagnetic and is only sensitive to fourth-order virtual hopping processes. In contrast, for a CT excited state where an unpaired spin on a metal site moves to the ligand site [Fig. 1(a)], the leading order contribution is sensitive to second-order virtual hopping processes owing to the Hund's coupling on the ligand site and is given by $J_{\mathrm{ex}} \propto-\left(t^{2} / \Delta_{\mathrm{CT}}^{2}-J_{H}^{2}\right) J_{H}$. Since $\Delta_{\mathrm{CT}}$ can be several times larger than both $t$ and $J_{H}$ in these materials, one can in principle transiently enhance $J_{\text {ex }}$ by exciting a CT transition with an ultrafast laser pulse.

Currently, there are several experimental approaches to measuring ultrafast laser induced exchange $\left(\delta J_{\mathrm{ex}}\right)$ modification. One is to detect $\delta J_{\mathrm{ex}}$ induced coherent spin precession using $\mathrm{THz}$ emission [2] or magneto-optical Kerr spectroscopy [10]. Another is to resolve the transient renormalization of spin wave energies or exchange splitting energies using techniques such as femtosecond stimulated Raman scattering [3] or photoemission spectroscopy $[20,21]$, respectively. Yet another is to measure the effects of $\delta J_{\text {ex }}$ on nonlinear responses such as the magneto-refractive effect [22] or magnetization-induced second harmonic generation [23,24]. We propose an alternative approach based on measuring $\delta J_{\mathrm{ex}}$ induced displacive excitation of coherent phonons, dubbed spin-DECP. In conventional DECP [25], an ultrafast optical pulse instantaneously modifies the charge distribution in a material, which shifts the atomic coordinates of the potential energy minimum, thereby producing a restoring Coulombic force $\vec{F}_{C}$ that initiates oscillatory lattice motion. In a superexchange system, the equilibrium metal-ligand-metal bonding angle $\theta$ is determined by a balance between the total elastic and total magnetic energies. The latter typically has the form $\sum_{i j} J_{\text {ex }}(\theta)\left\langle\vec{S}_{i} \cdot \vec{S}_{j}\right\rangle$, where $\left\langle\vec{S}_{i} \cdot \vec{S}_{j}\right\rangle$ is the spin correlator between metal sites $i$ and $j$. An instantaneous change in magnetic energy due to $\delta J_{\mathrm{ex}}$ shifts the potential energy minimum away from the equilibrium $\theta$ value, producing a restoring exchange force $\vec{F}_{\text {ex }}$ that initiates oscillatory motion [Fig. 1(b)]. The advantage of using spin-DECP to detect $\delta J_{\text {ex }}$ over the aforementioned techniques is that it relies only on the existence of short-range $\left(\left\langle\vec{S}_{i} \cdot \vec{S}_{j}\right\rangle \neq 0\right)$ and not longrange $\left(\left\langle\vec{S}_{i}\right\rangle \neq 0\right)$ magnetic correlations. This helps to disentangle $\delta J_{\mathrm{ex}}$ from the many laser-induced processes that are known to affect magnetic order [26], and it can be applied to both antiferromagnetic (AFM) and ferromagnetic (FM) materials.

An ideal material for demonstrating ultrafast ligand-tometal CT induced enhancement of $J_{\mathrm{ex}}$ using spin-DECP is $\mathrm{CrSiTe}_{3}$, a prototypical superexchange $\mathrm{FM}$ insulator with a layered honeycomb structure. In this material, the magnetic $(S=3 / 2)$ degrees of freedom originate from half-filled $\mathrm{Cr}$ (metal) $3 d t_{2 g}$ orbitals, which interact predominantly with their nearest neighbors [27] within the honeycomb layer via FM superexchange mediated by the Te (ligand) $5 p$ orbitals. Direct AFM exchange between $\mathrm{Cr} t_{2 g}$ orbitals also exists but is expected to be weak due to the large $\mathrm{Cr}-\mathrm{Cr}$ distance [28]. Owing to its quasi-two-dimensional structure, short-range intralayer FM correlations in $\mathrm{CrSiTe}_{3}$ persist up to $\sim 110 \mathrm{~K}[27,29]$, far above the Curie temperature $T_{c}=33 \mathrm{~K}$. Based on optical absorption [28] and density functional theory (DFT) calculations [30,31], optical excitation across the direct band gap of $\mathrm{CrSiTe}_{3}$ primarily involves CT from the Te $p$ to $\mathrm{Cr} e_{g}$ orbital [Fig. 2(a)], which, within our single $d$-orbital model, potentially leads to a $\sim 5$ fold enhancement of $J_{\text {ex }}$ using reported values of $\Delta_{\mathrm{CT}} \sim 1 \mathrm{eV}$ and $t \sim 0.5 \mathrm{eV}$ for $\mathrm{CrSiTe}_{3}$ [31] and a typical value of $J_{H} \sim 0.5 \mathrm{eV}$ [32]. The multi $d$-orbital nature of the
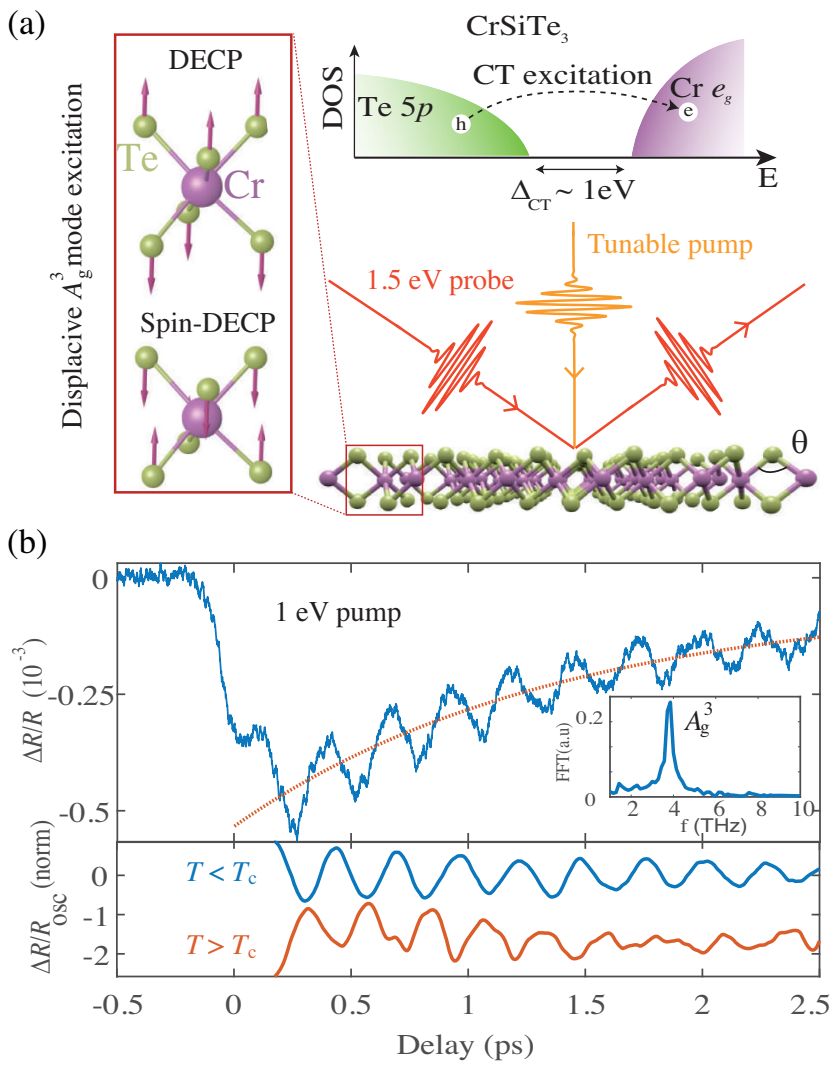

FIG. 2. Spin-DECP detection method in $\mathrm{CrSiTe}_{3}$. (a) Schematic of $\mathrm{CrSiTe}_{3}$ density of states (DOS) showing that excitation with $1 \mathrm{eV}$ light creates a hole in the Te $5 p$ valence band and an electron in the $\mathrm{Cr} e_{g}$ conduction band. This excitation has the dual effect of reducing the electrostatic attraction between the $\mathrm{Cr}$ and Te ions and also enhancing $J_{\text {ex }}$, which acts to decrease and increase the $\mathrm{Cr}-\mathrm{Te}-\mathrm{Cr}$ bond angle $\theta$, respectively, in turn launching the $A_{g}^{3}$ phonon mode with opposite initial directions as shown in the left panel. A schematic of the pump-probe optical reflectivity experiment used to detect the phase of the coherent $A_{g}^{3}$ mode is shown below. (b) (Top) Transient reflectivity of $\mathrm{CrSiTe}_{3}$ following a $1 \mathrm{eV}$ pump pulse taken at $T=25 \mathrm{~K}$ (pulse duration $100 \mathrm{fs}$, pump fluence $0.25 \mathrm{~mJ} / \mathrm{cm}^{2}$, sampling interval $0.2 \mathrm{fs}$ [14]). Inset: Fourier transform of the data with a clear peak at the $A_{g}^{3}$ mode frequency. (Bottom) Oscillatory component of the reflectivity transients $\left(\Delta R / R_{\mathrm{osc}}\right)$ taken at $T=25 \mathrm{~K}$ (blue) and $T=125 \mathrm{~K}$ (orange, vertically offset for clarity) obtained by subtracting a fitted exponential background (dashed orange line in top panel) from the raw data and then applying a moving average filter. 
magnetism in $\mathrm{CrSiTe}_{3}$ does not alter the conclusions drawn from our single $d$-orbital model [Fig. 1(a)] because the energies of the additional second-order virtual hopping processes introduced do not depend on the relative spin on the $\mathrm{Cr}$ sites [14]. We note that CT excitation is also expected to weaken the AFM direct exchange, thus further enhancing the net FM exchange. This is because Coulomb repulsion from an additional electron in the $e_{g}$ orbital suppresses virtual hopping between $\mathrm{Cr} t_{2 g}$ orbitals, and because virtual hopping from a half-filled to empty $e_{g}$ orbital gives rise to an FM exchange according to the Goodenough-Kanamori rules [33-35].

To distinguish spin-DECP from conventional DECP effects, which is generally challenging because both contribute to exciting a coherent phonon [11], we target modes whose oscillation phase is sensitive to the excitation mechanism. An example is the $A_{g}^{3}$ optical phonon mode in $\mathrm{CrSiTe}_{3}$, which involves the periodic expansion and contraction of the Te octahedra that surround each $\mathrm{Cr}$ ion [Fig. 2(a)]. An optical excitation that transfers an electron from the $\mathrm{Te}^{2-}$ to $\mathrm{Cr}^{3+}$ ions launches this mode through DECP, primarily by reducing the electrostatic attraction between them, producing a repulsive $\vec{F}_{C}$ that causes an initial expansion of the Te octahedra [14]. Importantly, the same CT excitation also increases $J_{\text {ex }}$, which launches the mode through spin-DECP by pushing $\theta$ toward $90^{\circ}$ where the FM superexchange is strongest according to the Goodenough-Kanamori rules [33-35]. Since $\theta<90^{\circ}$ in equilibrium [36], this produces an attractive $\vec{F}_{\text {ex }}$ that causes an initial contraction of the Te octahedra. Thus, DECP and spin-DECP drive the $A_{g}^{3}$ mode with opposite initial phase. We note that any contribution from impulsive heating effects would act in phase with DECP [14].

Phase-resolved coherent phonon spectroscopy of bulk $\mathrm{CrSiTe}_{3}$ single crystals was performed using ultrafast pumpprobe optical reflectivity measurements [Fig. 2(a)] [14]. A typical reflectivity transient acquired using a pump photon energy resonant with $\Delta_{\mathrm{CT}}$ is shown in Fig. 2(b), revealing an abrupt drop at delay time $t=0$, followed by an exponential recovery on a $\tau \sim 2$ ps timescale, tracking the generation and relaxation of CT excitations, respectively. The periodic modulations atop the recovery arise from a coherent optical phonon with frequency $f \sim 3.8 \mathrm{THz}$, which can be assigned to the $A_{g}^{3}$ mode based on Raman spectroscopy and DFT calculations $[28,37,38]$. Since the change in reflectivity is linearly proportional to the atomic displacement coordinate $\Delta Q$ through the Raman tensor $(d \chi / d Q) \Delta Q$, where $\chi$ is the linear electric susceptibility, our measurements can distinguish between an initial increase versus a decrease of $\theta$. A comparison of the oscillatory component $\left(\Delta R / R_{\mathrm{osc}}\right)$, isolated by subtracting the exponential background from the data, acquired above and below $T_{c}$ reveal a $\pi$ phase difference [Fig. 2(b)]. This suggests that $A_{g}^{3}$ mode excitation evolves from conventional DECP to spin-DECP dominated upon cooling.

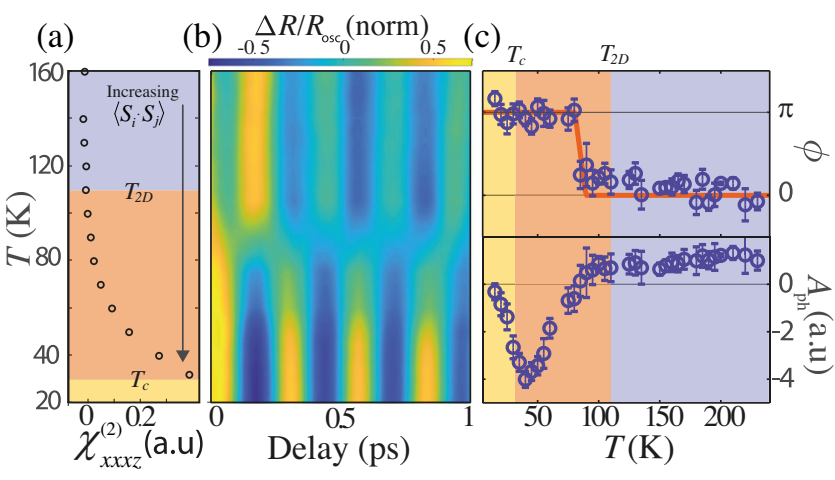

FIG. 3. CT excitation of $A_{g}^{3}$ mode. (a) Temperature dependence of the $x x x z$ component of the electric quadrupole SHG response of $\mathrm{CrSiTe}_{3}$, which is known to track the in-plane spin correlator $\left\langle\vec{S}_{i} \cdot \vec{S}_{j}\right\rangle$ [29]. Spin correlations markedly increase below $T_{2 D} \sim$ $110 \mathrm{~K}$ and then diverge at $T_{c}=33 \mathrm{~K}$ when long-range order sets in. (b) Temperature dependence of the normalized oscillatory component of the reflectivity transients $\left(\Delta R / R_{\mathrm{osc}}\right)$ taken with $1 \mathrm{eV}$ pump excitation after application of a moving average filter. (c) Temperature dependence of the $A_{g}^{3}$ phonon oscillation phase (top) and amplitude (bottom) extracted from the $\left(\Delta R / R_{\text {osc }}\right)$ data. The former was extracted through fitting [14] and the red line is a guide to the eye. The latter was extracted from the FFT amplitude, with a positive or negative sign denoting a 0 or $\pi$ oscillation phase, respectively. Error bars on the phase and amplitude data are estimated based on the accuracy of determining peak locations in the $\Delta R / R$ data and the FFT spectra, respectively.

To verify a spin-DECP mechanism at low temperature in $\mathrm{CrSiTe}_{3}$, we note that pronounced growth of 2D FM correlations was detected below a temperature scale $T_{2 \mathrm{D}} \approx 110 \mathrm{~K}$ by both neutron scattering [27] and optical second harmonic generation [29]. Therefore, one should expect spin-DECP effects to be present well above $T_{c}$. A complete temperature dependence of $\Delta R / R_{\text {osc }}$ and the optical second harmonic susceptibility of our sample are displayed in Figs. 3(a) and 3(b). The phonon oscillation phase $\phi$ extracted from the $\Delta R / R_{\text {osc }}$ data [14] undergoes a sharp change from 0 to $\pi$ below $T \sim 90 \mathrm{~K}$ [Fig. 3(c)], well inside the $2 \mathrm{D}$ correlated paramagnetic regime. To understand this behavior, we reanalyze the data with $\phi$ absorbed into the sign of the oscillation amplitude $A_{\mathrm{ph}}$. This procedure is valid because $\phi$ must be either 0 or $\pi$, corresponding to positive and negative signs for $A_{\mathrm{ph}}$, and helps visualize shifts in the relative weight of the DECP and spin-DECP contributions. As shown in Fig. 3(c), $A_{\mathrm{ph}}$ is positive $(\phi=0)$ and temperature independent for $T>T_{2 \mathrm{D}}$, consistent with the $A_{g}^{3}$ mode being driven exclusively by DECP. Below $T_{2 \mathrm{D}}$ where $\left\langle\vec{S}_{i} \cdot \vec{S}_{j}\right\rangle$ begins to grow, a spinDECP contribution with negative amplitude $(\phi=\pi)$ starts to compete with $\mathrm{DECP}$, causing $A_{\mathrm{ph}}$ to decrease and eventually change sign at $T \sim 90 \mathrm{~K}$ where $\vec{F}_{\text {ex }}$ becomes equal and opposite to $\vec{F}_{C}$ [14]. This crossover temperature has no measurable pump fluence dependence as expected since both the total $\vec{F}_{\text {ex }}$ and $\vec{F}_{C}$ should scale with the CT 
excitation density. As a control, we also measured coherent oscillations of an $E_{g}$ phonon using time-resolved second harmonic generation [39] under identical experimental conditions. In contrast to the $A_{g}^{3}$ mode, the $E_{g}$ mode can only be excited via impulsive stimulated Raman scattering and not conventional or spin-DECP due to symmetry considerations. We verified that neither the amplitude nor phase of the $E_{g}$ mode exhibit the aforementioned temperature dependence [14].

Below $90 \mathrm{~K}, A_{\mathrm{ph}}$ becomes increasingly negative as $\left\langle\vec{S}_{i} \cdot \vec{S}_{j}\right\rangle$ continues to rise [Fig. 3(a)]. However $A_{\text {ph }}$ reaches a minimum around $T_{c}$ and then turns back toward zero upon further cooling despite $\left\langle\vec{S}_{i} \cdot \vec{S}_{j}\right\rangle$ continuing to increase. This trend can be explained by a weakening of the spin-DECP contribution due to exchange striction below $T_{c}$. Previous x-ray diffraction studies on $\mathrm{CrSiTe}_{3}$ [36] showed that below $T_{c}$ there is a continual change in lattice parameters that brings $\theta$ closer to $90^{\circ}$. This means that the separation in $\theta$ between the potential energy minima of the equilibrium and CT states becomes smaller [Fig. 1(b)], progressively reducing $\vec{F}_{\text {ex }}$. Exchange striction must dominate the competing effect of increasing $\left\langle\vec{S}_{i} \cdot \vec{S}_{j}\right\rangle$, leading to a net decreasing spinDECP to DECP ratio upon cooling below $T_{c}$.

To uniquely demonstrate that the observed ultrafast enhancement of $J_{\mathrm{ex}}$ is induced by a ligand-to-metal CT transition, we repeated our coherent phonon spectroscopy measurements with a pump photon energy of $0.14 \mathrm{eV}$, far below the indirect $(\sim 0.4 \mathrm{eV})$ band gap of $\mathrm{CrSiTe}_{3}$ [28], to suppress CT transitions between the Te and $\mathrm{Cr}$ ions. We note that the absorption edge of $\mathrm{CrSiTe}_{3}$ is characterized by a long Urbach tail [40] that extends well below the indirect gap [28], allowing for finite absorption even at $0.14 \mathrm{eV}$. Given that the highest energy phonon mode in $\mathrm{CrSiTe}_{3}$ lies at $0.06 \mathrm{eV}$ [28], absorption at such low photon energies is likely to be dominated by transitions into midgap defect states [41] rather than phonon assisted CT processes. In fact, DFT calculations have shown that it is energetically favorable to form $\mathrm{Cr} / \mathrm{Si}$ anti-site defects in $\mathrm{CrSiTe}_{3}$ [41] and scanning tunneling microscopy measurements on the closely related compound $\mathrm{CrGeTe}_{3}$ have shown that this type of defect produces a finite density of in-gap states [42]. An optical CT excitation from the Te $p$ to Si defect orbitals should produce a Coulombic force between the $\mathrm{Te}$ and $\mathrm{Cr}$ atoms in the same direction as in the $\mathrm{Te}-\mathrm{Cr} \mathrm{CT}$ state due to increased charge negativity of the $\mathrm{Te}$ site. However, because the spin of the photohole on the Te site is independent of the spin on the $\mathrm{Cr}$ sites, second-order virtual hopping processes in the Te-Si CT state do not contribute to $J_{\mathrm{ex}}$. In line with this scenario, Fig. 4 shows that a $0.14 \mathrm{eV}$ pump is indeed able to excite the $A_{g}^{3}$ mode well above $T_{c}$ via conventional DECP but that no phase change occurs down to $7 \mathrm{~K}$ [14]. We note that, while a renormalization of $J_{\mathrm{ex}}$ imparted by a photo-assisted virtual hopping (i.e., Floquet engineering) mechanism can in principle also drive spin-DECP, such effects are negligible

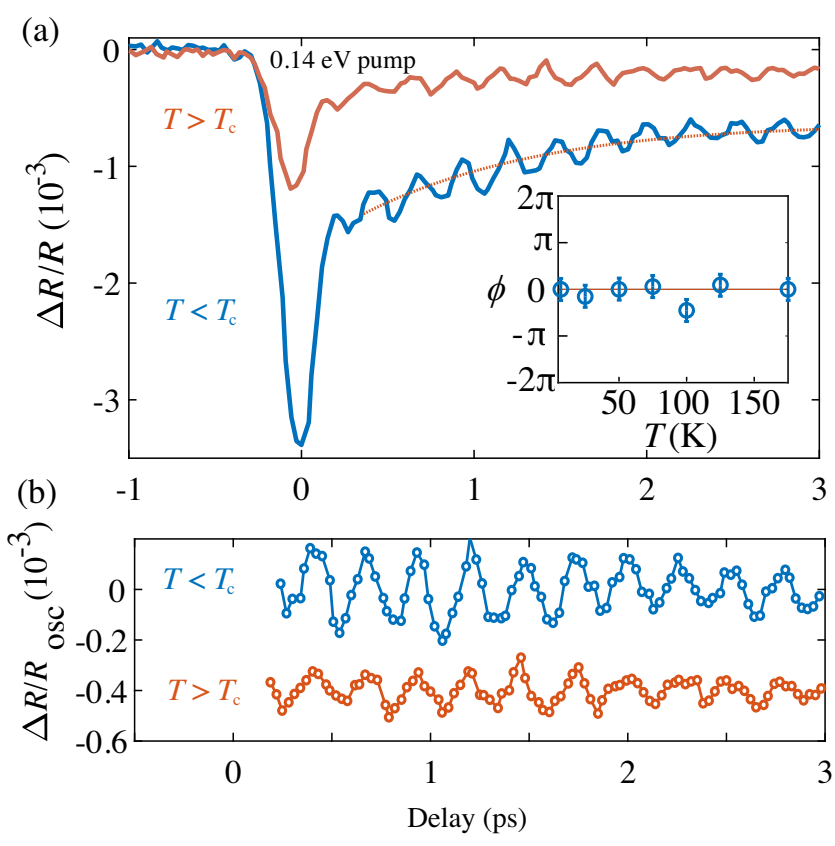

FIG. 4. Subgap excitation of $A_{g}^{3}$ mode. (a) Transient reflectivity of $\mathrm{CrSiTe}_{3}$ following a $0.14 \mathrm{eV}$ pump pulse (pulse duration $100 \mathrm{fs}$, pump fluence $8 \mathrm{~mJ} / \mathrm{cm}^{2}$, sampling interval $30 \mathrm{fs}$ [14]) taken at $T=25 \mathrm{~K}$ (blue) and $T=100 \mathrm{~K}$ (orange) showing oscillations at the $A_{g}^{3}$ mode frequency. Inset: Temperature dependence of the $A_{g}^{3}$ phonon oscillation phase extracted using the same method as described for the $1 \mathrm{eV}$ pump case. Error bars are estimated based on the accuracy of determining peak locations in the $\Delta R / R$ data. (b) Oscillatory component of the reflectivity transients shown in panel (a) obtained by subtracting a fitted exponential background [dashed orange line in panel (a)] from the raw data. Orange curve is vertically offset for clarity.

$(<1 \%)$ for the pump electric fields $(\sim 0.1 \mathrm{~V} / \AA)$ used in our experiments [14].

The ability to significantly enhance nearest-neighbor superexchange via ligand-to-metal CT excitation as we have demonstrated can potentially be harnessed to transiently manipulate magnetic materials in new ways. Examples include optically inducing FM order above $T_{c}$, switching between FM and AFM ordering patterns by altering the ratio of nearest-neighbor to further neighbor interactions, which are known to compete, for example, in transition-metal trichalcogenide materials such as $\mathrm{CrSiTe}_{3}$ $[43,44]$, or inducing order in geometrically frustrated magnets by controlling the light polarization to generate anisotropic changes in $J_{\mathrm{ex}}$ that relieve frustration. Optically induced FM ordering above $T_{c}$ was not achievable in our experiments because the stabilization of long-range FM ordering in $\mathrm{CrSiTe}_{3}$ depends predominantly on interlayer exchange [27,29], which is not enhanced by our pump. Moreover, owing to the intra- and interlayer exchange timescales in $\mathrm{CrSiTe}_{3}\left(h / J_{\text {ex }} \sim 4 \mathrm{ps}\right)$ being longer than the electron-hole recombination time $[\tau<2$ ps according to Fig. 2(b)], there is likely insufficient time for global spin rearrangement. Realizing the aforementioned vision will 
therefore involve targeting exchange pathways in materials that most strongly affect magnetic order, engineering both materials and excitation protocols to extend $\tau J_{\text {ex }} / h$, and developing ab initio approaches to calculate $J_{\text {ex }}$ in the CT state of complex solids to refine the molecular toy model estimates considered in this Letter.

This work was supported by ARO MURI Grant No. W911NF-16-1-0361. D. H. and G. R. also acknowledge support from the David and Lucile Packard Foundation. D. H. acknowledges support for instrumentation from the Institute for Quantum Information and Matter, an NSF Physics Frontiers Center (PHY-1733907). A. R. acknowledges support from the Caltech Prize Fellowship, the Zuckerman Foundation, and the Israel Science Foundation (Grant No. 1017/20). The MRL Shared Experimental Facilities are supported by the MRSEC Program of the NSF under Grant No. DMR 1720256, a member of the NSF-funded Materials Research Facilities Network. S. D. W. acknowledges support from the Nanostructures Cleanroom Facility at the California NanoSystems Institute (CNSI). G. R. also acknowledges partial support through DOE Award No. DE-SC0019166.

*Corresponding author.

dhsieh@caltech.edu

[1] J. Mentink, K. Balzer, and M. Eckstein, Nat. Commun. 6, 6708 (2015).

[2] R. Mikhaylovskiy, E. Hendry, A. Secchi, J. H. Mentink, M. Eckstein, A. Wu, R. Pisarev, V. Kruglyak, M. Katsnelson, T. Rasing et al., Nat. Commun. 6, 8190 (2015).

[3] G. Batignani, D. Bossini, N. Di Palo, C. Ferrante, E. Pontecorvo, G. Cerullo, A. Kimel, and T. Scopigno, Nat. Photonics 9, 506 (2015).

[4] S. Owerre, J. Phys. Commun. 1, 021001 (2017).

[5] M. Claassen, H.-C. Jiang, B. Moritz, and T. P. Devereaux, Nat. Commun. 8, 1192 (2017).

[6] G. Batignani, E. Pontecorvo, D. Bossini, C. Ferrante, G. Fumero, G. Cerullo, S. Mukamel, and T. Scopigno, Ann. Phys. (Amsterdam) 531, 1900439 (2019).

[7] J. Liu, K. Hejazi, and L. Balents, Phys. Rev. Lett. 121, 107201 (2018).

[8] S. Chaudhary, D. Hsieh, and G. Refael, Phys. Rev. B 100, 220403(R) (2019).

[9] J. M. Losada, A. Brataas, and A. Qaiumzadeh, Phys. Rev. B 100, 060410(R) (2019).

[10] H. B. Zhao, D. Talbayev, X. Ma, Y. H. Ren, A. Venimadhav, Q. Li, and G. Lüpke, Phys. Rev. Lett. 107, 207205 (2011).

[11] S. Wall, D. Prabhakaran, A. T. Boothroyd, and A. Cavalleri, Phys. Rev. Lett. 103, 097402 (2009).

[12] I. Handayani, R. Tobey, J. Janusonis, D. Mazurenko, N. Mufti, A. Nugroho, M. Tjia, T. Palstra, and P. Van Loosdrecht, J. Phys. Condens. Matter 25, 116007 (2013).

[13] D. Talbayev, J. Lee, S. A. Trugman, C. L. Zhang, S.-W. Cheong, R. D. Averitt, A. J. Taylor, and R. P. Prasankumar, Phys. Rev. B 91, 064420 (2015).
[14] See Supplemental Material, which includes Refs. [9,15-19], at http://link.aps.org/supplemental/10.1103/PhysRevLett .125 .197203 for additional details about the experiment and theoretical models.

[15] M. Liebel, C. Schnedermann, T. Wende, and P. Kukura, J. Phys. Chem. A 119, 9506 (2015).

[16] G. Batignani, C. Ferrante, G. Fumero, and T. Scopigno, J. Phys. Chem. Lett. 10, 7789 (2019).

[17] E. Pavarini, E. Koch, F. Anders, and M. Jarrell, Correlated Electrons: From Models to Materials (Forschungszentrum Jülich, Zentralbibliothek, Verlag, 2012).

[18] J. C. Slater, Phys. Rev. 36, 57 (1930).

[19] M.-C. Lee et al., Phys. Rev. B 99, 144306 (2019).

[20] H.-S. Rhie, H. A. Dürr, and W. Eberhardt, Phys. Rev. Lett. 90, 247201 (2003).

[21] R. Carley, K. Döbrich, B. Frietsch, C. Gahl, M. Teichmann, O. Schwarzkopf, P. Wernet, and M. Weinelt, Phys. Rev. Lett. 109, 057401 (2012).

[22] R. R. Subkhangulov, A. B. Henriques, P. H. O. Rappl, E. Abramof, T. Rasing, and A. V. Kimel, Sci. Rep. 4, 4368 (2014).

[23] A. Melnikov, I. Radu, U. Bovensiepen, O. Krupin, K. Starke, E. Matthias, and M. Wolf, Phys. Rev. Lett. 91, 227403 (2003).

[24] M. Matsubara, A. Schroer, A. Schmehl, A. Melville, C. Becher, M. Trujillo-Martinez, D. G. Schlom, J. Mannhart, J. Kroha, and M. Fiebig, Nat. Commun. 6, 6724 (2015).

[25] H. J. Zeiger, J. Vidal, T. K. Cheng, E. P. Ippen, G. Dresselhaus, and M.S. Dresselhaus, Phys. Rev. B 45, 768 (1992).

[26] A. Kirilyuk, A. V. Kimel, and T. Rasing, Rev. Mod. Phys. 82, 2731 (2010).

[27] T. J. Williams, A. A. Aczel, M. D. Lumsden, S. E. Nagler, M. B. Stone, J.-Q. Yan, and D. Mandrus, Phys. Rev. B 92, 144404 (2015).

[28] L. Casto, A. Clune, M. Yokosuk, J. Musfeldt, T. Williams, H. Zhuang, M.-W. Lin, K. Xiao, R. Hennig, B. Sales et al., APL Mater. 3, 041515 (2015).

[29] A. Ron, E. Zoghlin, L. Balents, S. Wilson, and D. Hsieh, Nat. Commun. 10, 1654 (2019).

[30] X. Chen, J. Qi, and D. Shi, Phys. Lett. A 379, 60 (2015).

[31] S. Kang, S. Kang, and J. Yu, J. Electron. Mater. 48, 1441 (2019).

[32] M. Wu, Z. Li, T. Cao, and S. G. Louie, Nat. Commun. 10, 2371 (2019).

[33] J. B. Goodenough, Phys. Rev. 100, 564 (1955).

[34] J. B. Goodenough, J. Phys. Chem. Solids 6, 287 (1958).

[35] J. Kanamori, J. Phys. Chem. Solids 10, 87 (1959).

[36] V. Carteaux, G. Ouvrard, J. Grenier, and Y. Laligant, J. Magn. Magn. Mater. 94, 127 (1991).

[37] M.-W. Lin, H. L. Zhuang, J. Yan, T.Z. Ward, A. A. Puretzky, C. M. Rouleau, Z. Gai, L. Liang, V. Meunier, B. G. Sumpter et al., J. Mater. Chem. C 4, 315 (2016).

[38] A. Milosavljević, A. Šolajić, J. Pešić, Y. Liu, C. Petrovic, N. Lazarević, and Z. V. Popović, Phys. Rev. B 98, 104306 (2018).

[39] Y.-M. Chang, L. Xu, and H. W. K. Tom, Phys. Rev. B 59, 12220 (1999). 
[40] M. Kurik, Phys. Status Solidi (a) 8, 9 (1971).

[41] H. Cheng, J. Zhou, M. Yang, L. Shen, J. Linghu, Q. Wu, P. Qian, and Y. P. Feng, J. Mater. Chem. C 6, 8435 (2018).

[42] Z. Hao, H. Li, S. Zhang, X. Li, G. Lin, X. Luo, Y. Sun, Z. Liu, and Y. Wang, Sci. Bull. 63, 825 (2018).
[43] N. Sivadas, M. W. Daniels, R. H. Swendsen, S. Okamoto, and D. Xiao, Phys. Rev. B 91, 235425 (2015).

[44] B. L. Chittari, Y. Park, D. Lee, M. Han, A. H. MacDonald, E. Hwang, and J. Jung, Phys. Rev. B 94, 184428 (2016). 